Paper

\title{
Demonstrations of Polarization Imaging Capability and Novel Functionality of Polarization-Analyzing CMOS Image Sensor with 65 nm Standard CMOS Process
}

\author{
Takashi Tokuda ${ }^{\dagger}$ (member), Kiyotaka Sasagawa ${ }^{\dagger}$ (member), \\ Norimitsu Wakama ${ }^{\dagger}$ (student member), Toshihiko Noda ${ }^{\dagger}$, \\ Kiyomi Kakiuchi ${ }^{\dagger}$ and Jun Ohta ${ }^{\dagger}$ (fellow)
}

\begin{abstract}
A polarization-analyzing CMOS image sensor with $65 \mathrm{~nm}$ standard fabrication process was designed and characterized. Polarization-analyzing image sensor pixel was realized using wire grid structures designed with a metal wiring layer within the standard CMOS process. Taking advantage of sub-100 nm CMOS process, a fine grid pitch was realized. Polarization-analyzing performance significantly higher than our previous sensors with $0.35 \mu \mathrm{m}$ CMOS process was obtained. Polarization imaging capability was demonstrated for a scene with local polarization variation. With an aim of further performance improvement, subtraction readout scheme and multiple layer stacked on-pixel polarizer were proposed and discussed.
\end{abstract}

Keywords: Polarization imaging, CMOS image sensor, $65 \mathrm{~nm}$ process, MEMS, $\mu$ TAS.

\section{Introduction}

Polarization sensing is one of the important optical measurement scheme used in various scientific and industrial applications. For instance, an optical rotation measurement is widely used to measure chirality of chemical species. The polarization of light is modulated when the light propagates the optically active material such as saccharide or protein. Polarization rotation reflects concentration and chirality of the measured materials. Not only as a single-beam measurement, but also in imaging, the polarization detection is expected to be an effective functional extension. Polarization mapping in captured images provides information of imaged objects such as reflectance, roughness, and relative angle of planes. Various technical approaches have been proposed and demonstrated to realize semiconductor photosensor and CMOS image sensor ${ }^{1)-7)}$.

In order to realize polarization-analyzing CMOS image sensors, we have proposed and demonstrated a design strategy to use monolithically embedded polarizer within standard CMOS processes ${ }^{8)-13)}$. Fig. 1 shows concept of our works. Wire grid structure made of metal wiring

\footnotetext{
Received September 25, 2013; Revised November 27, 2013; Accepted January 10, 2014

$\dagger$ Graduate School of Materials Science, Nara Institute of Science and Technology

(8916-5, Takayama-cho, Ikoma, Nara, 630-0192, Japan)
}

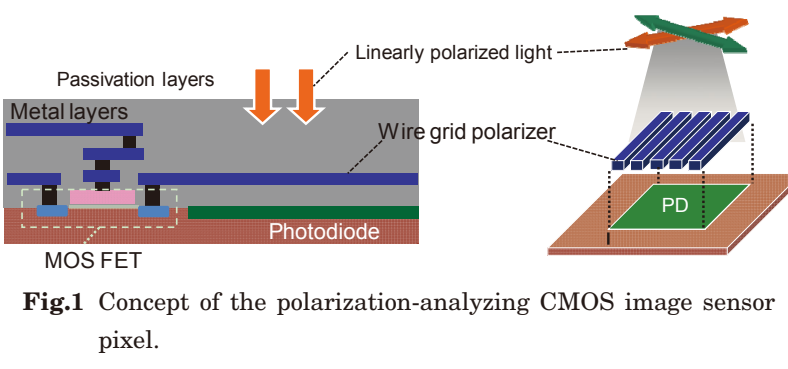

layer in CMOS process were integrated on photodiodes of image sensor pixels. We demonstrated that the wire grid structure works as an on-pixel polarizer.

This approach has technical advantages such as;

1. Since the polarizers are integrated in the CMOS fabrication process, no post-process is required to realize polarization detection function.

2. On-chip polarizers are accurately aligned to the pixel array, and it guarantees the angular datum.

3. Flexible pixel-by-pixel polarizer angle variation is available.

In our previous works, taking the advantage 3 , we designed an array of polarization-analyzing pixels with different polarizer angles (from 0 to $179 \mathrm{deg}$., in $1 \mathrm{deg}$. step) with $0.35 \mu \mathrm{m}$ standard CMOS process ${ }^{8)-9)}$. Combined with a linearly polarized light source with fixed polarization angle, we can obtain a polarization profile covering the whole angle in single frame measurement, without rotating the polarization of 
incident light.

From a viewpoint of single-pixel performance, polarization separation capability of single pixel is not very high (an extinction ratio less than 10$)^{8}$. It is due to large line / space dimensions that are comparable with the wavelength of light. However, we demonstrated that highly accurate polarization detection is available using a fitting-based estimation scheme using the whole angular polarization profile ${ }^{9)}$.

We also demonstrated the application feasibility of the polarization-analyzing CMOS image sensor with 0.35 $\mu \mathrm{m}$ process. An in-line dual-functional optical analyzer for chemical solution that is capable of measuring optical rotation and single-wavelength optical absorption was fabricated and demonstrated ${ }^{10)}$.

The most significant limitation of the previously reported polarization-analyzing CMOS image sensor fabricated with $0.35 \mu \mathrm{m}$ process is a requirement for uniformity of the light across the pixel array. For the profile-based estimation scheme, the measurement light beam should cover the size of the multi-angle polarization-analyzing pixel array $(400 \mu \mathrm{m} \times 400 \mu \mathrm{m})$. It also means that the sensor is basically applicable only for polarization measurement, and polarization imaging in which we see polarization angle in pixel-level resolution is not available. In order to overcome this limitation and extend the application of the polarizationanalyzing CMOS image sensor, an improvement of pixellevel polarization separation performance is necessary.

Considering the basic electromagnetic theory, the straightforward way to improve the extinction ratio is to reduce the pitch of the wire grid structure. We have reported that polarization-analyzing active pixel sensor TEGs (test element groups) fabricated with $65 \mathrm{~nm}$ standard CMOS process have a significantly higher polarization separation performance ${ }^{11}$. Based on this result, in this work, we designed and characterized the performance of a polarization-analyzing CMOS image sensor using $65 \mathrm{~nm}$ standard CMOS process. We describe the design and functionality of the sensor, including limitations due to the image sensor design with sub-100nm standard CMOS process. We also tried another design strategy to further improve the polarization separation performance. A series of active pixel sensor TEGs with stacked on-pixel polarizers were designed and characterized.

\section{Design of Polarization-Analyzing CMOS Image Sensor Using 65 nm Process}

\subsection{Simulated Polarization-Analyzing Performance of the On-chip Wire Grid Structures}

Prior to the CMOS sensor design, we performed numerical simulations to estimate expected performance of the polarization-analyzing CMOS image sensor with sub-100 nm CMOS process ${ }^{11)}$. The structure of on-pixel wire grid polarizer was simplified into a model structure with uniform cross section that is periodic in one dimension, as shown in an inset of Fig. 2. Due to the symmetry and periodicity, we used rigorous coupledwave analysis (RCWA) scheme and estimated the transmittance / reflectance of the plane light wave incidents into the grid structure.

Dimensions, materials, and electromagnetic / optical properties were defined based on typical values for sub$100 \mathrm{~nm}$ process. Since the polarization-analyzing performance strongly depends on the grid pitch, we performed the simulation for the dimensions from line / space $=60 \mathrm{~nm} / 60 \mathrm{~nm}$ to $140 \mathrm{~nm} / 140 \mathrm{~nm}$.

Fig. 2 shows the simulated polarization-analyzing performance (extinction ratio) of the on-pixel wire grid polarizers. The extinction ratio was defined as the intensity ratio of the transmit intensities of plane light wave with electric field perpendicular (TM) and parallel (TE) to the grid structure. As shown in Fig. 2, the extinction ratio strongly depends on the grid pitch, and drastically increases as decreasing the grid pitch. The simulation suggests that an extinction larger than 10 is expected at $600 \sim 800 \mathrm{~nm}$ when the sensor is designed with $65 \mathrm{~nm}$ standard CMOS process, in which line / space narrower than $100 \mathrm{~nm} / 100 \mathrm{~nm}$ is typically available.

We also performed simulations for multiple layer stacked on-pixel polarizers. Fig. 3 shows simulated extinction ratios for (a) metal $1 \& 2$, and (b) metal $1 \& 3$

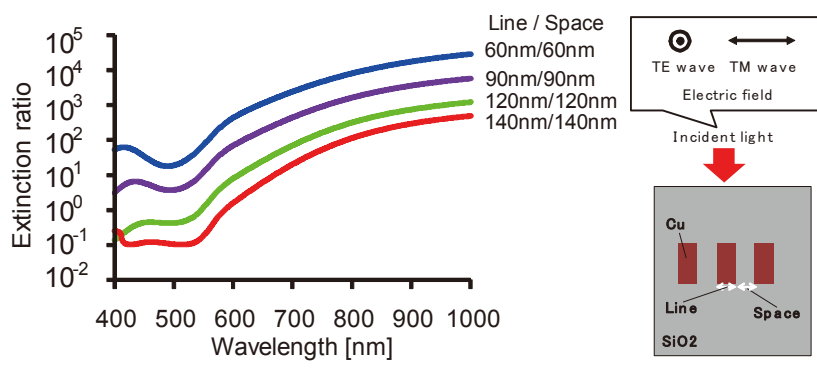

Fig.2 Simulated polarization-analyzing performance (extinction ratio) of on-pixel wire grid structures ${ }^{11}$. 


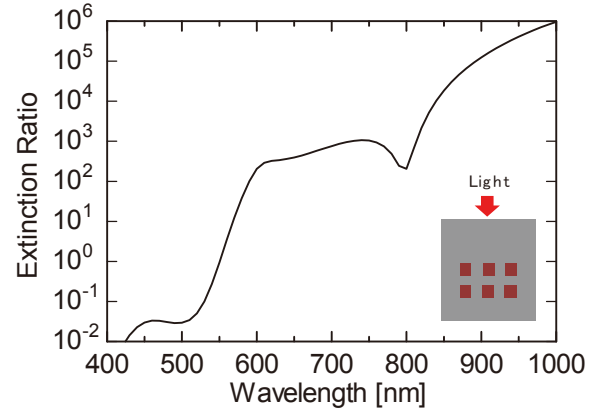

(a)

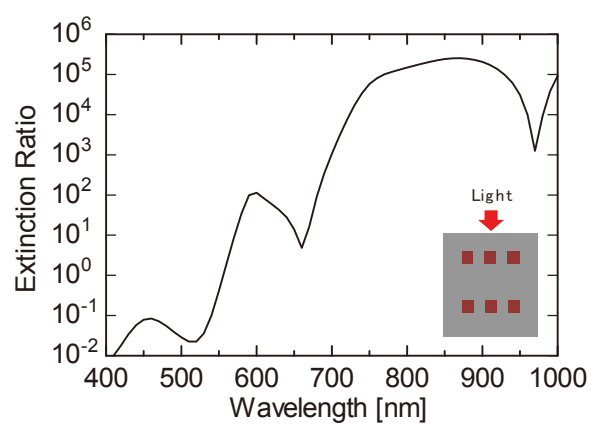

(b)

Fig.3 Simulated extinction ratios for (a) metal $1 \& 2$, and (b) metal $1 \& 3$ stacked on-pixel polarizers.

stacked on-pixel polarizers. The two stacked layers of polarizers with line / pitch $=130 \mathrm{~nm} / 130 \mathrm{~nm}$ were used as the model. In the model, two polarization grids were aligned coherently each other (no phase shift was introduced). The only difference between the models for Fig. 3 (a) and (b) is the vertical distance between two polarizer layers.

Fig. 3 suggests that, although the line $/$ space $=130$ $\mathrm{nm} / 130 \mathrm{~nm}$, the extinction ratio is larger than $20 \mathrm{~dB}$. The range of the extinction ratio is in a larger range than that for single layer polarizer with line / space of 90 $\mathrm{nm} / 90 \mathrm{~nm}$ in Fig. 2. Based on these simulations, we designed pixel TEGs with stacked on-pixel polarizers, as described in the next subsection.

\subsection{Design and Specifications of the Sensor}

We designed a polarization-analyzing CMOS sensor chip using a $65 \mathrm{~nm}$ standard CMOS process. The sensor chip consists of one polarization-analyzing image sensor and active pixel sensor TEGs. Fig. 4 shows (a) micrograph of the sensor chip and (b) layout of the polarization-analyzing pixel (for image sensor part). Table 1 shows specifications of the sensor.

The polarization-analyzing CMOS image sensor part consists of a $32 \times 32$ array of pixels with on-pixel polarizers. The pixel size is $20 \mu \mathrm{m} \times 20 \mu \mathrm{m}$ and fill factor of the pixel is as large as $43.6 \%$. For the on-chip polarizers, we used the minimum line / space widths available in the process. Due to a non-disclosure

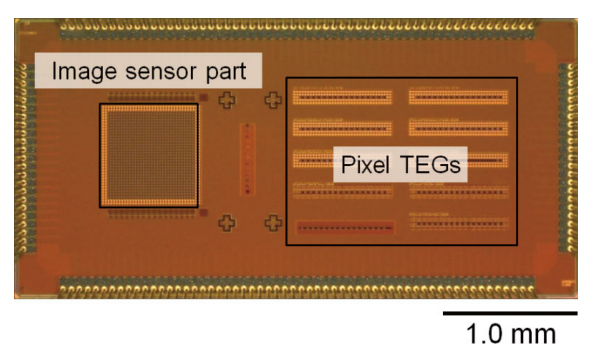

(a)

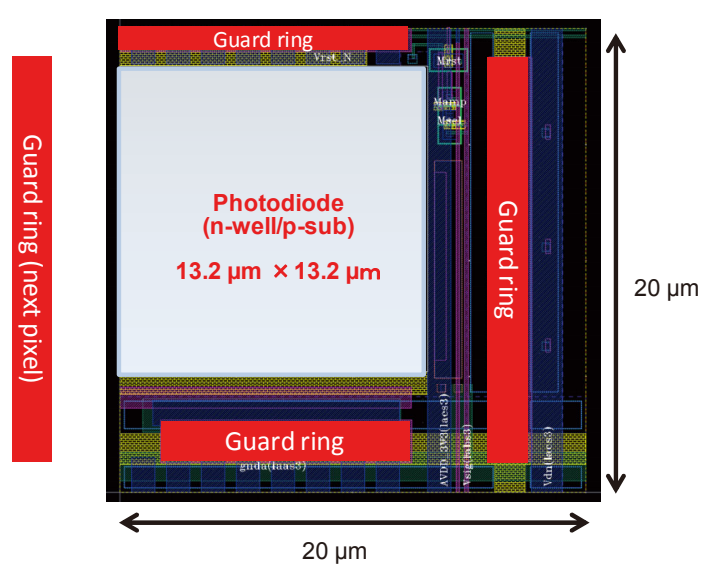

(b)

Fig.4 (a) micrograph of the sensor chip and (b) layout of the polarization-analyzing pixel (for image sensor part). Positions of guard ring structure ( $\mathrm{N}$-well carrier drain) are indicated with red rectangles.

Table 1 Specifications of the polarization-analyzing CMOS sensor chip.

\begin{tabular}{l|l}
\hline \hline \multicolumn{2}{c}{ Common specifications } \\
\hline Technology & $65 \mathrm{~nm}$ standard CMOS (Fujitsu) \\
\hline Photodiode & $\mathrm{N}$-well - P-sub \\
\hline Operation voltage & $3.3 \mathrm{~V}$ \\
\hline Chip size Polarization-analyzing image sensor part \\
\hline \multicolumn{2}{c}{$4.2 \mathrm{~mm} \times 2.1 \mathrm{~mm}$} \\
\hline Pixel number & $32 \times 32$ \\
\hline Pixel circuitry & 3 -Transistor active pixel sensor \\
\hline Pixel size & $20 \mu \mathrm{m} \times 20 \mu \mathrm{m}$ \\
\hline Fill factor & $43.6 \%$ \\
\hline On-pixel polarizer & Single layer (metal 1) \\
\hline \multicolumn{2}{c}{ Multiple stacked polarizer TEGs part } \\
\hline Pixel circuitry & 3 -Transistor active pixel sensor \\
\hline Pixel size & $20 \mu \mathrm{m} \times 20 \mu \mathrm{m}$ \\
\hline Fill factor & $56 \%$ \\
\hline On-pixel polarizer & Double layer (metal 1\&2, 1\&3), etc. \\
\hline \hline
\end{tabular}

agreement, exact dimensions of the wire grid structure cannot be shown in this paper. Differing from the 0.35 $\mu \mathrm{m}$ process that we used in the previous works ${ }^{8)-10)}$, any off-angle wire grid structure was allowed in the current $65 \mathrm{~nm}$ standard CMOS process. Thus, only wire grids with 0 deg. and 90 deg. are available with the $65 \mathrm{~nm}$ process. (45 deg. and $135 \mathrm{deg}$. lines are also allowed, but only for a larger grid pitch.) We implemented columns with 0 deg. and $90 \mathrm{deg}$. polarizers alternatively in the pixel array. Therefore, we have 16 columns with 0 deg. polarizer and 16 columns with $90 \mathrm{deg}$. polarizer.

The pixel circuit is 3 -transistor active pixel sensor, 


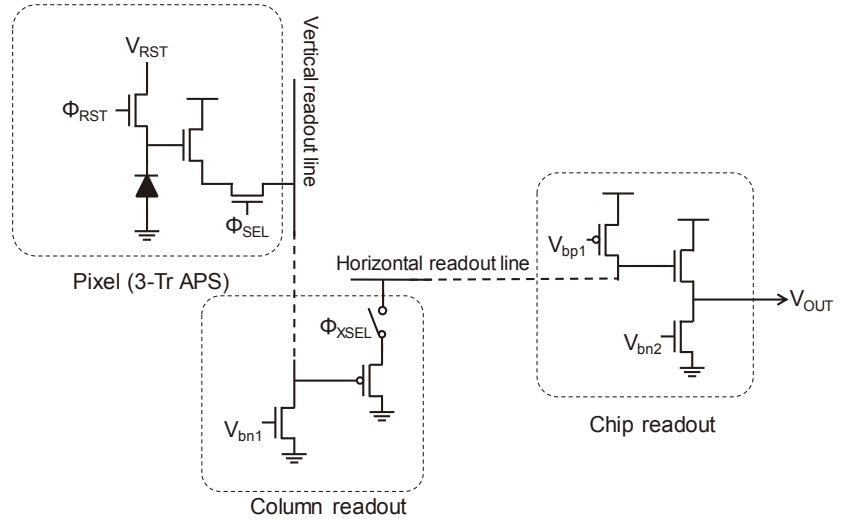

Fig.5 Analog circuit schematics for normal readout (image sensor part).

which is commonly used CMOS image sensor with standard CMOS process. Fig. 5 shows schematics of signal transfer circuitry from the pixel to the output. The pixel signal was transferred via a source follower chain from the pixel to the output buffer. The potential of the photodiode node in the pixel is read out as analog voltage and converted into digital signal by an A/D converter implemented on an interface board between the sensor and control PC.

In order to reduce inter-pixel crosstalk due to carrier diffusion from deep part of the sensor chip, we placed Nwell electron drain between the pixel photodiodes, as shown in Fig. 4 (b). A positive voltage is applied on these "guard ring" structure to collect diffused electron from the deep part of adjacent pixels. The effect of the guard ring voltage has been presented in ref. 13. In this work, we apply $3.3 \mathrm{~V}$ to obtain maximum electron collection effect.

As mentioned above, the full-angle polarizer variation is not available with the present fabrication processes. It means that fitting-based estimation using the full-angle polarization profile cannot be used for the present sensor. As an alternative polarization measurement scheme, we propose to use the subtraction measurement scheme. The idea was originally presented in our previous paper ${ }^{8)}$. For the subtraction measurement scheme, prior to the measurement, we prepare the situation in which the output from the pixels with $0 \mathrm{deg}$. and 90 deg. polarizers are equal. Starting with this situation, we observe the difference between the pixel signals with 0 deg. and $90 \mathrm{deg}$. polarizers. The change of the polarization angle can be detected as the increase / decrease of a difference between two pixels. For this purpose, in addition to the normal readout circuitry shown in Fig. 5, we implemented a subtraction readout circuitry. The pixel values of adjacent pixels are sampled

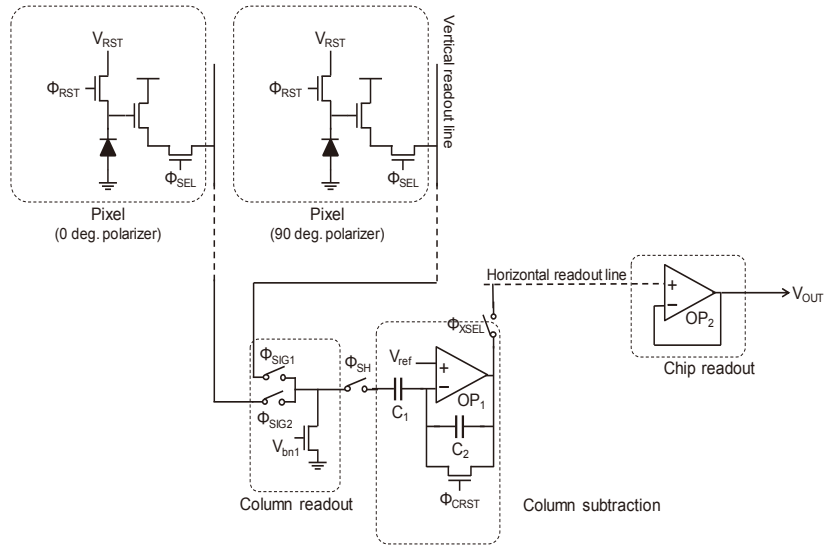

Fig.6 Readout circuitry for subtraction readout operation (image sensor part)

and compared at the columnar readout circuitry. The difference between adjacent pixels with 0 deg. and 90 deg. polarizers is read out as a measured value.

Fig. 6 shows the signal transfer circuitry for the differential measurement scheme. As same as the normal readout, the potential of the photodiode in the pixel is read out with source follower configured with the readout transistor in the pixel and current source transistor in the columnar readout circuit. The pixel signals read out by adjacent columnar readout circuits were sampled and compared by a column subtraction circuit. Difference of the signals from adjacent pixels (with 90 deg. polarizer and with 0 deg. polarizer) is read out via horizontal readout line and buffered by another analog buffer for output.

For the polarization-analyzing pixel TEG part, we also used active pixel sensor circuit with a size of $20 \mu \mathrm{m} \times 20$ $\mu \mathrm{m}$. However, for the sake of readout design, the layout is slightly modified and the fill factor is larger than that for image sensor pixel. Test pixels with different on-pixel polarizer structures were designed. Since we designed a large variation of TEGs, most of the test pixels were accessed via a selection circuitry such as a readout scanner.

\section{Performance of the Polarization- Analyzing CMOS Image Sensor}

\subsection{Polarization Separation Performance of Single-layer Polarizer in Pixel Array}

Fig. 7 shows the polarization profiles of the pixels with 0 deg. and 90 deg. on-pixel polarizers. In order to prevent crosstalk from adjacent pixels, linearly polarized light beam with a wavelength of $750 \mathrm{~nm}$ was focused onto the selected pixel. Profiles in Fig. 7 were measured by rotating the incident polarization as we reported 


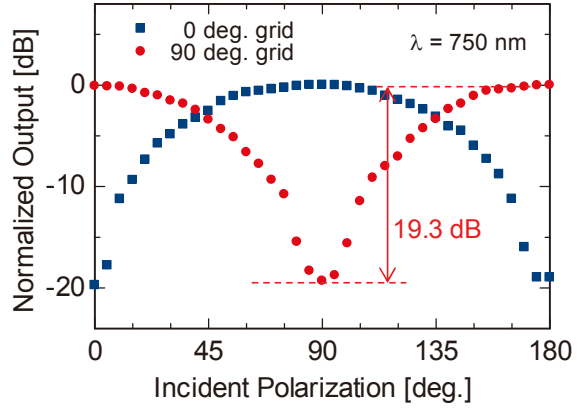

Fig.7 Single-pixel polarization separation performance ${ }^{12)}$.

previously ${ }^{12)}$. As shown in Fig. 7, the extinction ratio was as large as $19.3 \mathrm{~dB}$, that is significantly larger than that obtained with $0.35 \mu \mathrm{m}$ sensor $(3.3 \mathrm{~dB})^{9)}$. The value is consistent with the simulation (Fig. 2) and the extinction ratio for the single-pixel photo-sensor TEG ${ }^{11}$. This result means the polarization separation performance is kept intact when the pixel is used in a two dimensional pixel array. However, it should be taken into account that crosstalk between adjacent pixels is not negligible, as described in the next subsection.

\subsection{Crosstalk between Adjacent Pixels in a Pixel} Array

Introducing the pixel into an array, a crosstalk between neighboring pixels should be considered. In order to characterize the inter-pixel crosstalk, we performed a single-pixel illumination and measured the signal around the illuminated pixel ${ }^{13)}$. The light intensity observed in the adjacent un-illuminated pixel is understood as an artifact caused by the inter-pixel crosstalk.

Fig. 8 shows signal level of the inter-pixel crosstalk as a function of the pixel distance ${ }^{13)}$. The values were normalized with the signal when the pixel was directly illuminated. $3.3 \mathrm{~V}$ was applied on the guard ring surrounding the pixel photodiode (see Fig. 3 (b)). As shown in Fig. 8, the un-illuminated pixel next to the illuminated pixel shows a signal corresponding to approximately $3.0 \%(-15.0 \mathrm{~dB})$ of the illuminated pixel. The cross-talk to the second next pixel was as large as $0.5 \%$ of the illuminated pixel. This cross-talk signal depends on applied voltage on the guard ring. As measured and discussed in ref. 13, the guard ring voltage of $3.3 \mathrm{~V}$ (the condition for Fig. 8) gives the smallest inter-pixel crosstalk, which is approximately $28 \%$ smaller than the guard ring voltage of $0 \mathrm{~V}$.

The cross-talk signal from the nearest pixel is larger than the signal detected in the case the incident polarization is in complete crossed condition with the on-

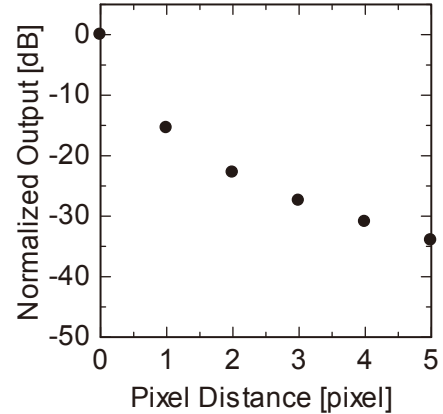

Fig.8 The artifact signal level caused by the inter-pixel crosstalk $^{13)}$.

pixel polarizer $(=-19.3 \mathrm{~dB}$, as shown in Fig. 6). Therefore, the net polarization-separation performance is limited to be as large as $15.0 \mathrm{~dB}$, for imaging mode in which the pixel array is illuminated with twodimensional light pattern.

\subsection{Polarization Imaging}

Although the inter-pixel crosstalk is not negligible, the polarization-separation performance of $15.0 \mathrm{~dB}$ is expected in imaging mode. We have reported that an image taken through an external polarizer shows a stripe-like feature depending on the relative angle between on-pixel polarizer and the external polarizer ${ }^{13)}$. In this work, to demonstrate the functionality of polarization imaging, image capturing experiment was performed for scenes with local polarization variation. Fig. 9 shows the experimental setup. Illumination from an LCD panel with rotating stand was used as uniformly polarized background. Three types of optical components were placed between the LCD panel and the polarization-analyzing CMOS image sensor. We captured images with a polarizing beam splitter that was aligned to transmit only 0 deg. polarization. We also used a $1 / 2 \lambda$ plate to obtain $90 \mathrm{deg}$. polarization rotation and a $1 / 4 \lambda$ plate to obtain linear to circular polarization conversion.

Fig. 10 shows images captured in the polarizationimaging experiments. Since columns with 0 deg. and 90 deg. on-pixel polarizers were aligned alternatively,

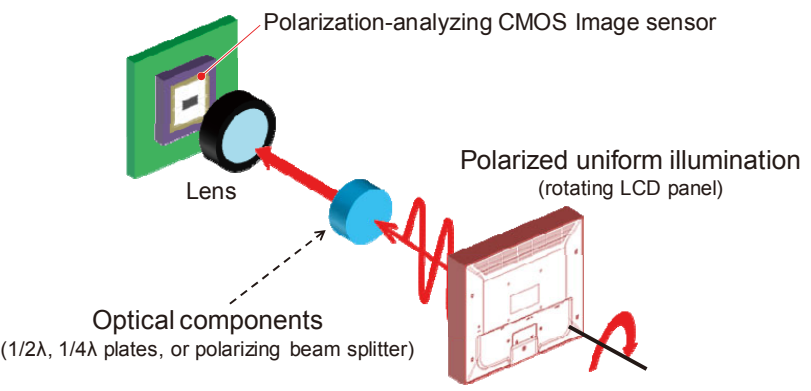

Fig.9 Experimental setup for the polarization imaging demonstration. 


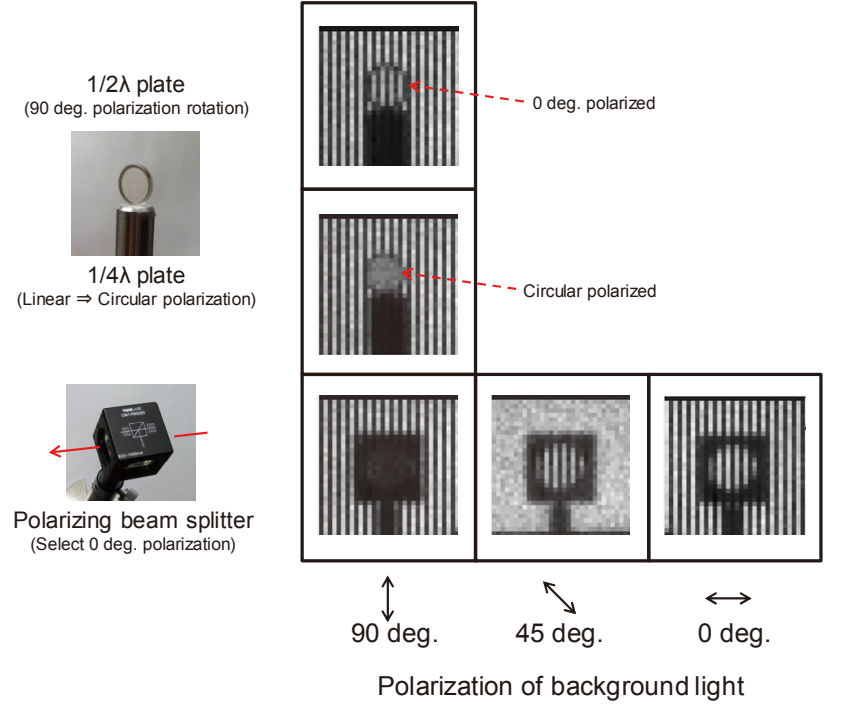

Fig.10 Captured images that have local polarization variation.

stripe features were observed if the imaging area was illuminated with linearly polarized light. Comparing the background of the images of polarizing beam splitter, we can see the polarization-selective sensitivity of the pixels and phase difference between $0 \mathrm{deg}$. and $90 \mathrm{deg}$ illuminations Since only 0 deg. polarization can be transmitted through the polarizing beam splitter, the pattern inside the beam splitter always shows the stripe feature with fixed phase. The brightness inside the splitter image depends on the portion of 0 deg. polarization included in the background light.

When the $1 / 2 \lambda$ plate was imaged, a clear phase shift of the stripe feature was observed due to the polarization rotation from 0 deg. +90 deg. For the $1 / 4 \lambda$ plate, the brightness inside the plate became uniform because of conversion from linear to circular polarization. All of the images shown in Fig. 10 show consistent features and suggest that the local variation of the polarization can be imaged by the present polarization-analyzing CMOS image sensor.

Not only these qualitative demonstration, as a future work, we expect to estimate the polarization angle with pixel-pair resolution, combining the subtraction mode read out described in the next section, or simply with an external numerical processing in PC.

\subsection{Subtraction Mode Readout}

Properties of the subtraction readout circuit were characterized. The sensor was illuminated with linearly polarized light and the output signal from the selected pixel pair was measured via the subtraction readout circuit. Fig. 11 shows the output of the subtraction readout circuit as a function of incident polarization. Characteristic curves obtained under weak and intense

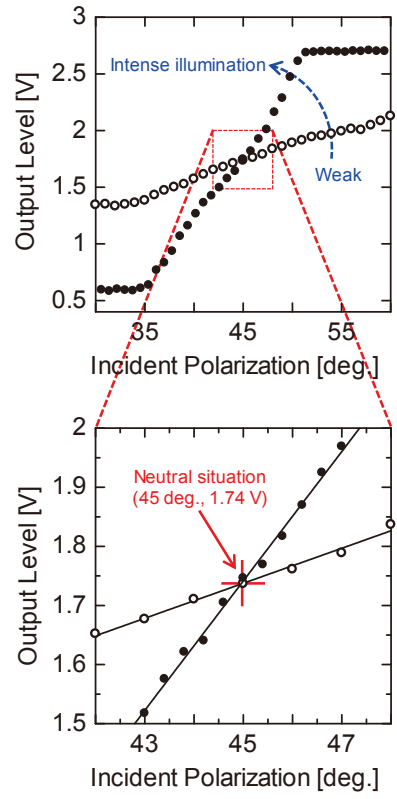

Fig.11 Output signal obtained via subtraction readout circuit.

illuminations are plotted.

The output signal of the subtraction readout circuit represents the difference between output levels from adjacent pixels with $0 \mathrm{deg}$. on-pixel polarizer and $90 \mathrm{deg}$. on-pixel polarizer. The output level also depends on the illumination intensity of the incident light, as shown in Fig. 11.

The output value obtained at $45 \mathrm{deg}$. polarization indicates that the output of the pixel with $0 \mathrm{deg}$. polarizer is equal to that of the pixel with $90 \mathrm{deg}$. polarizer, thus "neutral situation". As far as the output values from the pixels are within appropriate input range of the readout circuit, the "neutral situation" is independent from the illumination intensity. It means that we can reach the "neutral situation" by adjusting the incident polarization to obtain the output level of $1.74 \mathrm{~V}$, even with additional optical rotation factors between the linearly polarized light source and the sensor.

As mentioned in the section 2.2, we aim to use the subtraction readout for differential polarization measurement. The differential measurement can be a solution to observe relative change of polarization, such as an in situ optical rotation observation ${ }^{10)}$. In application, we will insert an optically active measurement target such as chiral solution in the light path. Prior to the in situ optical rotation observation, we rotate the incident polarization angle to obtain a calibration curve, and then, adjust the incident polarization angle to "neutral situation" for initialization. Observing the output level of the subtraction readout, we can track relative changes in 
the optical rotation of the measurement target. We must use the calibration curve for quantitative analysis.

In the present subtraction readout circuit, the magnification of the signal is defined with the fixed capacitances $\mathrm{C} 1$ and $\mathrm{C} 2$ in Fig. 5. Therefore, we prepare a measurement condition to obtain a favorable output profile by adjusting the illumination intensity and/or accumulation duration. As a further functional improvement, we expect that an implementation of gain selectivity with selectable C2 will provide an improved adaptability to various measurement situations.

\subsection{Performance Evaluation of Pixel TEGs with Multiple Layer Stacked Polarizers}

The polarization separation performance for test pixels with multiple layer stacked on-pixel polarizers was characterized. Fig. 12 shows measured extinction ratios obtained from test pixels with (a) metal $1 \& 2$, and (b) metal $1 \& 3$ stacked on-pixel polarizers. Due to NDA, the exact dimensions of line / space cannot be disclosed. We only mention that the multiple layer polarizers were designed with dimensions comparable with the model for the simulations (see Fig. 3). The values are larger than that for single-layer polarizer for the image sensor part.

The experimentally obtained extinction ratio for the multiple layer stacked polarizer was in comparable range $(10 \sim 20 \mathrm{~dB})$ with that for the single-layer polarizer (see Fig. 7). The maximum value was $19.2 \mathrm{~dB}$ that was obtained with the metal 1 \& 3 stacked polarizers at $800 \mathrm{~nm}$. Considering the line / space dimensions are larger for the stacked polarizers, this result suggests the effect of multiple layer structure.

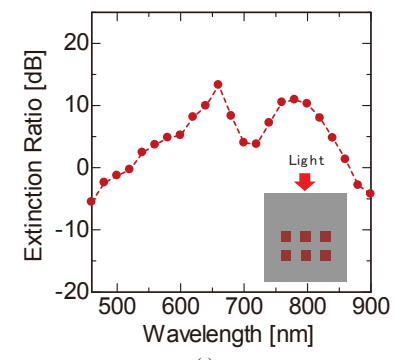

(a)

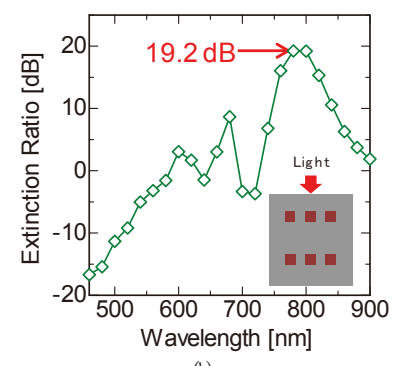

(b)

Fig.12 Extinction ratio for multiple layer stacked on-pixel polarizers.
However, the performance advantage of stacked polarizers was limited. As far as the extinction ratio is around $20 \mathrm{~dB}$, the single-layer on-pixel polarizer is still advantageous in transmittance and design flexibility.

Comparing with the simulated curves (Fig. 3), the experimentally obtained extinction ratio was smaller by one order or more. We consider this large discrepancy between simulated and measured performances originates in TM-TE crosstalk caused by light scattering due to pixel edges, which is not considered in the simulation. The extinction ratio was estimated by dividing the transmission for TM wave by transmission for TE wave. Theoretically, the extinction ratio is expected to be very large because TE wave transmission is almost zero in the simulated wavelength region. However, for the real pixel, pixel edges cause a small level of scattered light in TE incident configuration. It is detected as the TM-TE crosstalk. The TM-TE crosstalk causes an increase of the denominator of the extinction ratio, thus, a drastic decrease off the extinction ratio. This TM-TE crosstalk explains the limitation in the extinction ratio of the real pixels.

We also found that experimentally obtained spectral dependence shows additional features that are not exist in the simulated spectra (Fig. 3). For example, a dip of extinction ratio was observed around $700 \mathrm{~nm}$ in experimentally obtained spectra, as shown in Fig. 12 (a) and (b). This is because that the simulated model does not include passivation layers that cause a complicated multi-layer interference effect ${ }^{12)}{ }^{14}$ ). Since the dielectric passivation layers are expected to be optically inactive, the interference effect does not directly explain the spectral features in the experimental results. However, combined with the TM-TE crosstalk caused by pixel edges, it is reasonable to accept that the multi-layer interference affect the experimental results.

Based on these discussions, an introduction of nonuniform simulation scheme and model structure with pixel edge will be required for more accurate simulationbased polarizer design, especially for multiple layer stacked polarizers.

\section{Conclusions}

A polarization-analyzing CMOS image sensor with 65 nm standard CMOS process was designed and characterized. Compared with sensors fabricated with $0.35 \mu \mathrm{m}$ standard process, the pixel-level polarizationseparation performance was significantly improved, and an extinction ratio of $19.3 \mathrm{~dB}$ was obtained. Even 
considering inter-pixel crosstalk, a polarizationseparation performance of $15 \mathrm{~dB}$ can be expected in imaging operation The functionality of polarizationimaging and subtraction readout were demonstrated for the image sensor. We also discussed for further improvement of polarization separation performance with multiple layer stacked polarizers. Based on test pixel evaluation, it was confirmed that an extinction ratio around $20 \mathrm{~dB}$ can be achieved with stacked polarizer structure even taking relatively larger grid pitch than single-layer polarizer.

\section{Acknowledgements}

This work was partly supported in part by STARC through the "Silicon Implementation Support Program for Next Generation Semiconductor Circuit Architectures" sponsored by Japanese Ministry of Economy, Trade and Industry, by a Grant-in-Aid for Scientific Research \#24310101, by a Grant-in-Aid for Scientific Research on Innovative Areas ¥\#24106729 from the Ministry of Education, Science, Sports and Culture, Japan, and by the VLSI Design and Education Centre (VDEC), The University of Tokyo, in collaboration with Cadence Design Systems, Inc.

\section{References}

1) F. Boussaid, A. Bermak and V.G. Chigrinov: "Thin Photo-Patterned Micropolarizer Array for CMOS Image Sensors", IEEE Photonics Technology Letters, 21, 12, pp.805-807 ( June 2009)

2) M. Guillaumée, L.a. Dunbar, C. Santschi, E. Grenet, R. Eckert, O.J.F. Martin and R.P. Stanley: "Polarization sensitive silicon photodiodes using nanostructured metallic grids", Applied Physics Letters, 94, 19, p. 193503 (2009)

3) V. Gruev: "Fabrication of a dual-layer aluminum nanowires polarization filter array", Optics express, 19, 24, pp.24361-9 (Nov. 2011)

4) V. Gruev, J. Van der Spiegel and N. Engheta: "Dual-tier thin film polymer polarization imaging sensor", Optics express, 18, 18, pp.19292-303 (Aug. 2010)

5) V. Gruev, R. Perkins and T. York: "CCD polarization imaging sensor with aluminum nanowire optical filters", Optics express, 18, 18, pp.19087-94 (Aug. 2010)

6) M. Sarkar, D.S.S. San Segundo Bello, C. van Hoof and A. Theuwissen: "Integrated Polarization Analyzing CMOS Image Sensor for Material Classification", IEEE Sensors Journal, 11, 8, pp.1692-1703 (Aug. 2011)

7) S.B. Powell and V. Gruev: "Calibration methods for division-offocal-plane polarimeters", Optics Express, 21, 18, p. 21039 (Aug. 2013)

8) T. Tokuda, S. Sato, H. Yamada, K. Sasagawa and J. Ohta: "Polarisation-analysing CMOS photosensor with monolithically embedded wire grid polariser", Electronics Letters, 45, 4, p. 228 (2009)

9) T. Tokuda, H. Yamada, K. Sasagawa and J. Ohta: "PolarizationAnalyzing CMOS Image Sensor With Monolithically Embedded Polarizer for Microchemistry Systems", IEEE Transactions on Biomedical Circuits and Systems, 3, 5, pp.313-316 (May 2009)

10) T. Tokuda, H. Matsuoka, N. Tachikawa, N. Wakama, K. Terao, M.
Shibata, T. Noda, K. Sasagawa, Y. Nishiyama, K. Kakiuchi and J. Ohta: "CMOS sensor-based miniaturised in-line dual-functional optical analyser for high-speed, in situ chirality monitoring", Sensors and Actuators B: Chemical, 176, pp.1032-1037 (Jan. 2013) 11) S. Shishido, T. Noda, K. Sasagawa, T. Tokuda and J. Ohta: "Polarization Analyzing Image Sensor with On-Chip Metal Wire Grid Polarizer in 65-nm Standard Complementary Metal Oxide Semiconductor Process", Japanese Journal of Applied Physics, 50, pp.2-5 (2011)

12) K. Sasagawa, S. Shishido, K. Ando, H. Matsuoka, T. Noda, T. Tokuda, K. Kakiuchi and J. Ohta: "Image sensor pixel with on-chip high extinction ratio polarizer based on 65-nm standard CMOS technology", Optics Express, 21, 9, p. 11132 (Apr. 2013)

13) N. Wakama, D. Okabayashi, T. Noda, K. Sasagawa, T. Tokuda, K. Kakiuchi and J. Ohta: "Polarisation analysing complementary metal-oxide semiconductor image sensor in 65-nm standard CMOS technology", Journal of Engineering, 2013, pp.1-3 (Sep. 17, 2013)

14) M. Ikeda and Y. Kim: "Measurement and analysis on characteristics of transmission and polarization for $12 \mathrm{ML} 65 \mathrm{~nm}$ CMOS", Proceedings of 2010 IEEE Sensors, 2010, pp.548-551

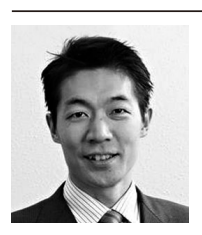

Takashi Tokuda received his B. E. and M. E. degrees in electronic engineering and $\mathrm{Ph}$. D. degree in materials engineering from Kyoto University, Kyoto, Japan, in 1993, 1995, and 1998, respectively. In 1999, he joined Graduate school of Materials Science, Nara Institute of Science and Technology (NAIST), Nara, Japan, as an Assistant Professor and was appointed as an Associate Professor in 2008.

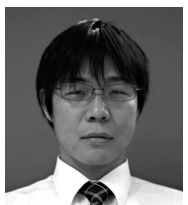

Kiyotaka Sasagawa received his B. S. degree from Kyoto University, Kyoto, Japan in 1999 and M. E. and Ph. D degrees in materials science from NAIST, in 2001 and 2004, respectively. He was a researcher in the National Institute of Information and Communications Technology from 2004 to 2008. Since 2008, he has been an assistant professor in Graduate school of Materials Science, NAIST.

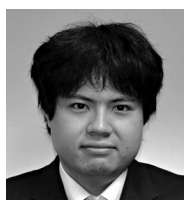

Norimitsu Wakama is a Ph. D. candidate in Graduate School of Materials Science, NAIST. He received his M. E. degree from Kansai University, JAPAN in 2011. His research focuses on the smart image sensors for biomedical applications.

Toshihiko Noda received his B.E. and M.E. degrees in electrical and electronic engineering in 2001, 2003, respectively, and Ph.D. degree in engineering in 2006, from Toyohashi University of Technology, Aichi, Japan. Since 2009, he has been an assistant professor in Graduate school of Materials Science, NAIST.

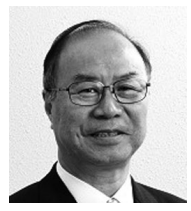

Kiyomi Kakiuchi received his B. S., M. S. and $\mathrm{Ph}$. D. degrees from Osaka University in 1977, 1979 , and 1982, respectively. Since 1997, He is a full professor in Graduate School of Materials Science, NAIST. His research focuses on the highly efficient chiral organic photo- chemistry. From 2009 to 2010, he was appointed as Vice-President of NAIST. Since 2013, he is Dean of Graduate School of Materials Science, NAIST.

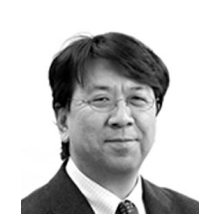

Jun Ohta received his B. E., M. E., and Ph. D. degrees in applied physics from the University of Tokyo, Japan, in 1981, 1983, and 1992, respectively. In 1983, he joined Mitsubishi Electric Corporation, Japan. From 1992 to 1993, he was a Visiting Researcher in University of Colorado, Boulder. In 1998, he joined the Graduate School of Materials Science, NAIST, as an Associate Professor, and was appointed as a full Professor in 2004. Dr. Ohta is a Fellow of ITE. 